\title{
APPLICATION OF WIRELESS SENSOR NETWORKS FOR AGRICULTURE PARAMETERS
}

\author{
AWATI J.S. ${ }^{1}$, PATIL V.S. ${ }^{2}$ AND AWATI S.B. ${ }^{3}$ \\ ${ }^{1}$ ETC department, RIT, Sakharale, Sangli, MS, India. \\ 2SCOE, Panhala, Kolhapur, MS, India. \\ 3DKTE, Ichalkarangi, Kolhapur, MS, India. \\ ${ }^{*}$ Corresponding Author: Email- jsa.awati@gmail.com
}

Received: January 30, 2012; Accepted: April 03, 2012

\begin{abstract}
In Today's world due to global warming and climate changes there is challenging situation in field of agriculture. Number of advanced methods and technologies are coming in agriculture to reduce cost and improve total productivity. Water is an important resource in agriculture. There is new concept of agriculture in controlled environment. To reduce other cost in agriculture i.e. soil testing. This paper concentrate on Measuring temperature, humidity, and controlling water supply depend on moisture content of soil.
\end{abstract}

Key words- humidity, WSN, soil, soil moisture, etc.

Citation: Awati J.S., Patil V.S. and Awati S.B. (2012) Application of Wireless Sensor Networks for Agriculture Parameters. International Journal of Agriculture Sciences, ISSN: 0975-3710 \& E-ISSN: 0975-9107, Volume 4, Issue 3, pp.- 213-215.

Copyright: Copyright@2012 Awati J.S, et al. This is an open-access article distributed under the terms of the Creative Commons Attribution License, which permits unrestricted use, distribution, and reproduction in any medium, provided the original author and source are credited.

\section{Introduction}

Agriculture is the backbone of the Indian economy. The development of agriculture in terms of area of land under cultivation, use of modern equipment and financial assistance to the farmers is absolutely essential. India is to progress economically with all sectors of the population enjoying its agricultural effect. One of the major problems present today is the less knowledge of the soil content \& types, less knowledge of the type of fertilizers to be added, the irrigation amount and pattern depending on the soil porosity and its water retention capacity. In the current Indian scenario analysis of soil to increase crop yields is not being used to a large extent primarily due to the cost involved and the inaccessibility of labs offering such testing facilities. Moreover due to small size of land holdings the procedure of sending soil samples to a far off lab and then taking decision does not seem economically viable.

The technological development in Wireless Sensor Networks made it possible to use in monitoring and control of Agriculture parameters in rural area. Due to uneven natural distribution of rain water it is very crucial for farmers to monitor and control the equal distribution of water to all crops in the whole farm or as per the requirement of the crop. There is no ideal irrigation method available which may be suitable for all weather conditions, soil structure and variety of crops cultures. It is observed that farmers have to bear huge financial loss because of wrong prediction of weather and incorrect irrigation method to crops. In this paper with the evolution in wireless sensor devices, it is possible to uses them for automatic environment monitoring and controlling the parameters of agriculture.

\section{Objective}

1. Study some of the agricultural parameters such as soil, Water, crops, Irrigation, chemical and fertilizers issues.

2. Study environmental issues of specific region.

3. Adopt technique for controlling the agricultural parameters: Use different sensors to sense the agricultural parameters.

- Collect and transmit the information using Embedded System.

- Using wireless receiver receives the information and does the needful controlling action.

- Design the module which useful in rural development.

- Obtain the readings, observations.

- Measure the performance and benefits to the farmers.

\section{Scope}

This paper would take the opportunity to design an instrument that is able to monitor the temperature soil and humidity of an agriculture environment. 
In this paper the potential of wireless sensor networks (WSNs) are explored in an original context, the small agriculture of rural area. The goal is to confront an emerging technology with a concrete problem of world-wide dimensions, the sustainability of farming for land-holders living in rural area.

This paper aims to demonstrate the technology of both wireless sensor network and data visualization with the basis for the critical problems, challenges and future goals of development and applications. And we propose a model integrated the two above technologies, emphasis on the processing.

\section{Wireless Sensor Networks}

A wireless sensor is a self-powered computing unit usually containing a processing unit, a transceiver and both analog and digital interfaces, to which a variety of sensing units - typically sampling physical data, such as temperature, humidity etc. can be adapted [2]. These sensors automatically organize themselves into an adhoc network, which means they do not need any preexisting infrastructure, as do cellular networks such as Zigbee is referred to such a network as an ad-hoc Wireless Sensor Network. Recently, WSNs have raised considerable interest in the computing and communication systems' research community. They have decisive advantages, compared with the technologies previously used to monitor environments via the collection of physical data. Whenever physical conditions change rapidly over space and time, WSNs allow for real-time processing at a minimal cost [3]. Their capacity to organize spontaneously in a network makes them easy to deploy, expand and maintain, as well as resilient to the failure of individual measurement points [4].

\section{Relative humidity}

Air, in our normal environment, always holds humidity. The number of water molecules in the air can vary substantially, e.g. it can be as dry as in a desert or as humid as in the tropics. There is an upper limit for the amount of humidity which air can hold at a given temperature. Beyond this limit saturation occurs. If for some reason the humidity level is pushed up to this limit, condensation occurs and fog or water droplets form. Relative humidity tells what percentage of this maximum amount of humidity is present in the air. In contrast to relative humidity, absolute humidity denotes the absolute amount of humidity in the air regardless of the saturation level expressed as the total mass of water molecules per air volume. The maximum possible amount of humidity as well as the actual present amount of humidity in the air is defined by so called water vapor pressures. According to Dalton's law, total air pressure is the sum of the partial vapor pressures of its components and water vapor pressure is one of them. The maximum amount of humidity, which air can hold, is defined by the so-called saturation water vapor pressure. This is a function of temperature.

$\mathrm{P}_{\text {water vapor }}=$ Water vapor pressure, $\mathrm{P}_{\text {oxygen }}=$ Oxygen pressure,

$\mathrm{P}_{\text {nitrogen }}=$ Nitrogen pressure, $\mathrm{P}_{\text {total }}=$ Total air pressure,

$\mathrm{P}_{\text {others }}=$ Other pressure

$\mathrm{P}_{\text {total }}=\mathrm{P}_{\text {water vapor }}+\mathrm{P}_{\text {oxygen }}+\mathrm{P}_{\text {nitrogen }}+\mathrm{P}_{\text {others }}$

Relative Humidity $(\%)=\mathrm{RH}(\%)=\left[\mathrm{P}_{\text {water vapor }} / \mathrm{P}_{\text {saturation }}\right]^{* 100}$

Soil- Soil is a natural body consisting of layers (soil horizon) of mineral constituents of variable thicknesses, which differ from the parent materials in their morphological, physical, chemical, and mineralogical characteristics. Soil is composed of particles of broken rock that have been altered by chemical and environmental processes that include weathering and erosion. Soil differs from its parent rock due to interactions between the lithosphere, hydrosphere, atmosphere, and the biosphere. It is a mixture of mineral and organic constituents that are in solid, gaseous and aqueous states. In Engineering, soil is referred to as regolith, or loose rock [5].

Soil pH- Soil pH is a measure of the acidity in soils. pH is defined as the negative logarithm (base 10) of the molar concentration of dissolved hydronium_ions $\left(\mathrm{H}_{3} \mathrm{O}^{+}\right)$. It ranges from 0 to 14 , with 0 being most acidic, 14 being highly basic, and 7 being neutral. Soil $\mathrm{pH}$ affects plant nutrient availability by controlling the chemical form of the nutrient. The preferred soil pH varies between plants and can be modified via various soil amendments.

Soil moisture- Soil moisture is the amount of water present in the soil. Gaps between soil particles are called pore spaces or voids. These voids contain various amounts of either water or air. Soil moisture content can be expressed in different basis:

- Gravimetric: the mass of water/mass of solid material

- Volumetric: the volume of soil/total porosity

The amount of void space within a soil depends on the distribution of particle sizes, and is quantified by soil porosity. Soil moisture is more generally considered within the context of hydrology, where it represents the immediate store of infiltrating rainfall, before it contributes to groundwater recharge [6].

Soil Condition Control- Soil water also affects the crop growth. Therefore, the monitor \& control of soil condition has a specific interest, because good condition of a soil may produce the proper yield. The proper irrigations and fertilizations of the crops are varies as per the type, age, phase and climate. The $\mathrm{pH}$ value, moisture contains, electric conductivity and the temp of a soil are some key parameters. The $\mathrm{pH}$ valves and other parameters will help to monitor the soil condition. The temperature and the moisture can be controlled by the irrigation techniques like drift and sprinkles system.

Humidity sensor- A number of different methods are used for measuring humidity in air. The choice of the most suitable method is usually made by the user based on the local situation. The use of a simple but correctly applied humidity measuring device often permits achieving a better accuracy or meeting the particular requirements.

\section{Main Interface Circuit}

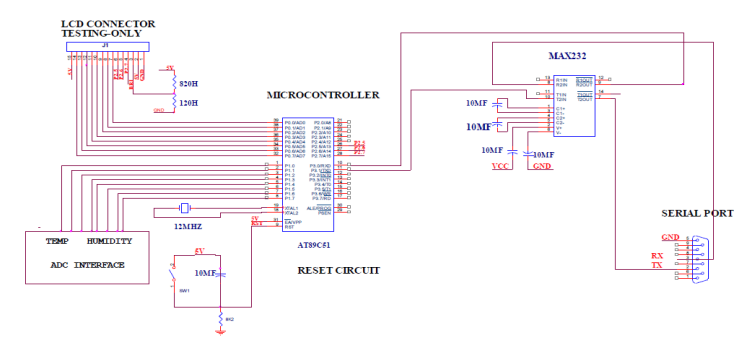




\section{Testing and Results}

Tests were done to verify the reliability and accuracy of the Temperature and Humidity monitoring system. Two different set of tests were conducted.

Test conducted in closed room- The location of the test was at our room. The windows and doors were all shut so as to have minimum air-flow from the outside environment. The purpose of the test was to find out how accurate is the Temperature and $\mathrm{Hu}$ midity monitoring system.
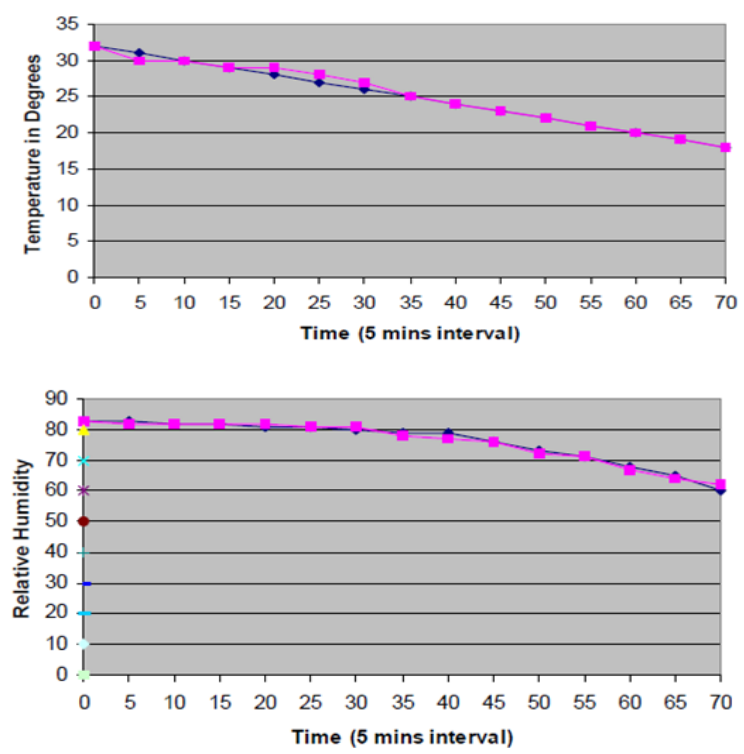

Test conducted with an open environment: The location of the test is at garden.
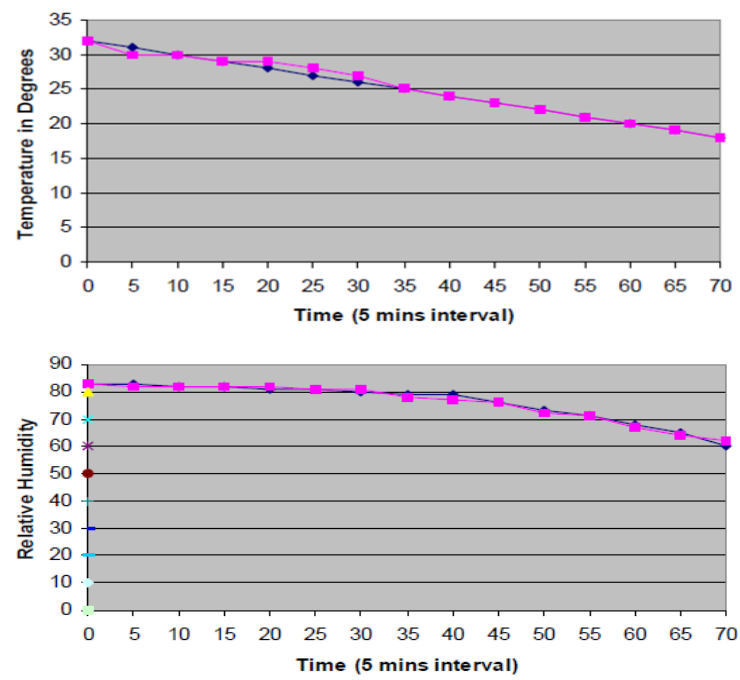

The blue line indicates- The open environment, The pink line indicates- The project prototype.

The second indicates that the results of the first test are quite accurate as the results are almost the same.

Test results-The test results show that both the two test conducted has similar test patterns. The project prototype has similar values after a prolonged time. This could be because it takes a longer period for the sensor to settle down.

\section{Conclusion}

The end result is a fully functional Temperature and Humidity Monitoring System, ready for use, from a personal point of view; the paper has been a fulfilling and rewarding experience. This paper deal it both technically and also in terms of how to manage and conduct agriculture parameters. This paper has put into practice a wide range of engineering skills to solve agriculture problems.

\section{References}

[1] Eiman Kanjo and Peter Landshoff (2007) IEEE Distributed Systems Online, Urban Computing and Mobile Devices, 8(7).

[2] Burri N., von Rickenbach P. and Wattenhofer R. Dozer (2007) ultra-low power data gathering in sensor networks, ACM, 450459.

[3] Barrenetxea G.,Ingelrest F.,Schaefer G.,Vetterli M.,Couach O.,Parlange M. (2008) SensorScope: Out-of-the-Box Environmental Monitoring, In The 7th International Conference on Information Processing in Sensor Networks (IPSN).

[4] Stoianov L., Nachman S., Madden Pipenet (2007) IEEE International Conference on Information Processing in Sensor Networks.

[5] Lemos S.G., Noguera A.R., Torre-Neto A., Parra A., Alonso J. (2007) Journal of Agricultural and Food Chemistry, 55 46584663.

[6] Chaudhary D.D., Nayse S.P., Waghmare L.M. (2011) International Journal of Wireless \& Mobile Networks, 3, 1.

[7] Zhi Sun and lan F. Akyildiz (2010) IEEE Secon, 978-1-4244$7151-5 / 10$. 